\title{
A SAÚDE INFANTIL E A VIA DE NASCIMENTO: UMA REVISÃO INTEGRATIVA
}

\section{CHILD HEALTH AND THE BIRTH PATH: AN INTEGRATIVE REVIEW}

\section{Daniela Sezílio Barreto ${ }^{1} *$ Lilian Fernandes Arial Ayres ${ }^{2 *} *$ Bruno David Henriques $^{3} *$ Gabriela Diniz $^{*}$ Pinto Coelho $^{4} *$ Mara Rúbia Maciel Cardoso do Prado ${ }^{5} *$ Tatiane Roseli Alves Castro $^{6}$}

\section{RESUMO}

Objetivo: identificar as evidências científicas existentes na literatura sobre o impacto da microbiota adquirida pelo recém-nascido na saúde infantil, conforme a via de nascimento. Métodos: trata-se de uma revisão integrativa. Utilizaram-se as bases de dados PubMed, LILACS, IBECS e CUMED. Para uma indexação mais eficiente das publicações, os filtros foram desenvolvidos para a busca em PubMed de acordo com o Medical Subject Headings. Para pesquisa na BVS, foram usados os Descritores em Ciência da Saúde. Os artigos foram analisados utilizando-se um instrumento de coleta de dados. Resultados: A amostra deste estudo constituiu-se de 21 trabalhos cujas publicações variaram de 2011 a 2018. Verificou-se que os Estados Unidos e a Dinamarca foram os locais com maior número de trabalhos publicados. Conclusão: Foram evidenciados fatores que podem interferir no desenvolvimento de doenças, na infância, em crianças nascidas por cesariana, como asma, dermatite, obesidade, doença intestinal, metabólica e até mesmo alguns tipos de cânceres.

Palavras-chave: Microbiota; Cesárea; Parto; Sistema Imunitário; Recém-Nascido.

\section{ABSTRACT}

Objective: to identify the scientific evidence in the literature on the impact of the microbiota acquired by the newborn on child health, according to the route of birth. Methods: this is an integrative review. The PubMed, LILACS, IBECS and CUMED databases were used. For a more efficient indexing of publications, the filters were developed for searching PubMed according to the Medical Subject Headings. For research in the VHL, the Health Science Descriptors were used. The articles were analyzed using a data collection instrument. Results: The sample of this study consisted of 21 works whose publications varied from 2011 to 2018. It was found that the United States and Denmark were the places with the largest number of published works. Conclusion: Factors that can interfere in the development of diseases in childhood were evidenced in children born by cesarean section, such as asthma, dermatitis, obesity, intestinal, metabolic disease and even some types of cancers.

Keywords: Microbiota; Cesarean Section; Parturition; Immune System; Infant, Newborn.

\footnotetext{
${ }^{1}$ Graduada em Enfermagem. Universidade Federal de Viçosa. E-mail: daniela.barreto@aiesec.net. ORCID: https://orcid.org/00000003-4588-0948

${ }^{2}$ Professora. Departamento de Medicina e Enfermagem. Universidade Federal de Viçosa. E-mail: lilian.ayres@ufv.br. ORCID: http://orcid.org/0000-0003-3809-2660

${ }^{3}$ Professor. Departamento de Medicina e Enfermagem. Universidade Federal de Viçosa. E-mail: bruno.david@ufv.br. ORCID: https://orcid.org/0000-0002-6844-6661

${ }^{4}$ Graduada em enfermagem. Universidade Federal de Viçosa. E-mail: gaabi.dpc@ hotmail.com. ORCID: http://orcid.org/0000-00020044-3579

${ }^{5}$ Professora. Departamento de Medicina e Enfermagem. Universidade Federal de Viçosa. E-mail: mara.prado@ufv.br ORCID: https://orcid.org/0000-0001-6314-0009

${ }^{6}$ Graduanda. Aluna do curso de Enfermagem. Universidade Federal de Viçosa. Pará. Brasil. E-mail: tatiaeracastro@gmail.com. ORCID: https://orcid.org/0000-0003-3668-8401
} 


\section{INTRODUÇÃO}

Os números de cesariana (CS) aumentaram nas últimas décadas. A taxa quase dobrou entre 2000 e 2015 e estimou-se que $21,1 \%$ dos nascimentos ocorreram por este procedimento. Existem disparidades entre os países que variam de 44,3\% na América Latina e Caribe para apenas $4,1 \%$ na África Ocidental e Central ${ }^{1}$. O Brasil possui um dos maiores percentuais de cesáreas quando comparado a outros países. Este aumento ocorreu mundialmente, sobretudo no Brasil, a partir da década de $1970^{2}$.

Segundo a Organização Mundial de Saúde (OMS), a cesariana não deve exceder os limites de $10 \%$ a $15 \%$ do total de partos e, quando forem maiores do que $10 \%$, não estão associadas à redução de mortalidade materna nem neonatal ${ }^{3}$. A taxa de cesárea no Brasil realizada pelo Sistema Único de Saúde (SUS) é de 40\%, já na saúde suplementar, fica em torno de $85 \%^{4,5}$. Foi constatado em um estudo que $93,8 \%$ das vias de nascimento na rede privada são por cesariana ${ }^{6}$. Tais dados elucidam a qualidade da atenção obstétrica no país.

A cesariana é uma operação segura quando realizada de acordo com a avaliação médica e é responsável pela redução de mortalidade materna e perinatal, porém é utilizada de forma errônea e sem critérios médicos, o que justifica suas altas taxas no Brasil ${ }^{4,7}$. Essa via de nascimento em gestantes de aproximadamente 37 semanas torna-se um fator de risco para o recémnascido (RN) e pode estar relacionada a diversos prejuízos para o mesmo, como o risco aumentado de doenças e de outros problemas, tais como: asma, gastroenterite, doença celíaca, atraso no início da amamentação, desconforto respiratório neonatal e maior taxa de internações em unidades de terapia intensiva ${ }^{4,8}$. Além da morbidade, a cesárea influencia diretamente na mortalidade materna e na neonatal, prejudica o vínculo da mãe ao recém-nascido, prejudica as futuras gestações e gera impactos na saúde da criança ${ }^{4,7}$.

Estudos apontam que um dos principais fatores que determina e que influencia a composição da microbiota desenvolvida pelo $\mathrm{RN}$ é a via de nascimento 7,9. Sabe-se que o parto vaginal fornece bactérias que são fundamentais para ajudar o bebê a desenvolver seu sistema imunológico e que, quando não é exposto a essas bacterias, ele pode ter sua imunidade prejudicada, aumentando a chance de ter alergias, leucemia, inflações, artrite juvenil, asma, obesidade, deficiências imunológicas e desenvolver um papel importante na etiologia da cárie dentária, entre outros ${ }^{8,10-12}$.

Percebe-se que a microbiota do RN é definida de acordo com a via de nascimento e isso influencia no desenvolvimento de doenças no neonato e na sua vida futura. A 
literatura brasileira possui poucos estudos que elucidam essa temática. Diante disso, este trabalho objetiva identificar as evidências científicas existentes na literatura sobre $o$ impacto da microbiota adquirida pelo $\mathrm{RN}$ na saúde infantil, conforme a via de nascimento.

\section{MÉTODOS}

Trata-se de uma revisão integrativa que sintetiza o conhecimento científico existente sobre a problemática que se deseja estudar. $\mathrm{O}$ pesquisador acompanha $\mathrm{o}$ desenvolvimento da temática no decorrer do tempo, gera conhecimentos e elabora novas teorías ${ }^{13}$.

As fases adotadas para a realização deste trabalho foram as propostas por Mendes, Silveira e Galvão, sendo elas: $1^{\circ}$ definição do tema e da pergunta da pesquisa; $2^{\circ}$ escolha dos critérios de inclusão e exclusão de trabalhos; $3^{\circ}$ identificação dos estudos selecionados; $4^{\circ}$ categorização dos estudos selecionados; $5^{\circ}$ análise e interpretação dos resultados; $6^{\circ}$ síntese do conhecimento e apresentação da revisão ${ }^{14}$.

A elaboração da questão norteadora foi realizada utilizando-se a estratégia PICO: Paciente, Intervenção, Comparação e "Outcomes" (desfecho). Em conjunto, esses componentes possuem grande relevância para o planejamento da pergunta de pesquisa e, por conseguinte, para a busca de evidências científicas ${ }^{14}$. O presente estudo tem como pergunta norteadora: qual é a produção de conhecimento sobre o impacto da microbiota adquirida pelo RN na saúde infantil conforme a via de nascimento? Sendo o primeiro elemento, (P), o $\quad \mathrm{RN}, \quad \mathrm{o}$ segundo (I) corresponde a cesariana, o terceiro, $(\mathrm{P})$, é o parto vaginal e o quarto, $(\mathrm{O})$, impacto da microbiota adquirida pelo $\mathrm{RN}$ na saúde infantil.

A definição dos critérios de inclusão de trabalhos para a realização desta pesquisa foram artigos disponíveis na íntegra, publicados em português, inglês ou espanhol, nos quais o tema da questão norteadora tenha sido contemplado, e com até dez anos de publicação. Os critérios de exclusão foram revisões, cartas, editoriais, opinião de especialistas, estudos feitos com prematuros e os que não abordavam assuntos relacionados à via de nascimento.

A identifcação dos artigos selecionados nesta revisão foi a partir de uma busca avançada em bases de dados eletrônicas, como Sistema Online de Busca e Análise de Literatura Médica (MEDLINE) (via Pubmed), Literatura Latino-americana e do Caribe em Ciências da Saúde (LILACS), Índice Bibliográfico Español en Ciencias de la Salud (IBECS) (via Biblioteca Virtual em Saúde- BVS), no dia 31 de agosto de 2020. Teve como estratégia de busca, a seleção de trabalhos baseados nos filtros da pesquisa, conforme o quadro 1. 
Quadro 1- Processo de busca de publicações de acordo com as bases de dados.

\begin{tabular}{|c|c|c|c|}
\hline $\begin{array}{l}\text { Base de } \\
\text { dados }\end{array}$ & Filtro de pesquisa & $\begin{array}{c}\mathbf{N}^{\circ} \text { de } \\
\text { publicações }\end{array}$ & $\mathbf{N}^{\circ}$ de duplicatas \\
\hline PUBMED & $\begin{array}{c}\text { (((MICROBIOTA[MeSH Terms]) AND (Delivery, } \\
\text { Obstetric OR Cesarean Section OR Parturition[MeSH } \\
\text { Terms])) AND HUMAN[MeSH Terms]) NOT MILK, } \\
\text { HUMAN[MeSH Terms] }\end{array}$ & 206 & \multirow{2}{*}{$\begin{array}{c}54 \\
\text { DUPLICADOS }\end{array}$} \\
\hline BVS & $\begin{array}{c}\text { (tw:(MICROBIOTA)) AND (tw:(HUMANS)) AND } \\
\text { (tw:(PARTURITION OR CESAREAN SECTION OR } \\
\text { DEVILERY, OBSTETRIC)) AND NOT } \\
\text { (tw:(HUMAN,MILK)) }\end{array}$ & 119 & \\
\hline
\end{tabular}

Fonte: Os autores

Após o agrupamento das publicações encontradas nas duas bases eletrônicas, as duplicadas foram separadas, e os seguintes itens foram comparados: títulos, autores, ano e país. Após esse procedimento, foram mantidos na base os artigos com maior número de referências encontradas (PubMed). Dois autores, de forma independente, realizaram uma triagem dos títulos e dos resumos guiados pelos critérios de elegibilidade.

Em seguida, todos os artigos escolhidos foram obtidos na íntegra para nova seleção. Os pesquisadores examinaram, independentemente, todas as publicações para selecionar os estudos que preenchiam os critérios de inclusão para a revisão. As divergências foram resolvidas em consenso.

A seguir, os artigos foram analisados utilizando-se um instrumento de coleta de dados contendo: título do artigo e/ou do periódico, nome dos autores, país, idioma, ano de publicação, instituição do estudo, tipo de revista científica, características metodológicas dos estudos e avaliação do rigor metodológico, e adaptações para o assunto dessa revisão ${ }^{15}$. Essas informações foram inseridas no programa Excel.

As publicações também foram analisadas, segundo o nível de evidência, que são organizados em sete níveis, sendo eles: Nível I - revisão sistemática ou meta-análise de ensaios clínicos randomizados controlados ou de diretrizes baseadas em revisões sistemáticas de ensaios clínicos randomizados controlados; Nível II - evidências oriundas de pelo menos um ensaio clínico randomizado controlado e bem delineado; Nível III - estudo controlado sem randomização; Nível IV trabalhos provenientes de estudos bem desenhados como caso-controle ou coorte; Nível V - estudos provenientes de uma revisão sistemática de trabalhos qualitativos e descritivos; Nível VI - evidências de um único estudo descritivo ou qualitativo; Nível VII - evidências provenientes da opinião de 
autoridades e/ou relatórios de comitês de especialistas. Posteriormente à identificação das publicações selecionadas, os artigos foram analisados rigorosamente, interpretados e sintetizados com uma apresentação da revisão e a constituição das categorias.

\section{RESULTADOS}

A amostra deste estudo constituiu-se de 13 trabalhos. Os processos de busca, de exclusão e o número de artigos selecionados foram descritos passo a passo em um fluxograma (Figura 1).

Figura 1: Processo de seleção de artigos. Viçosa, 2020.

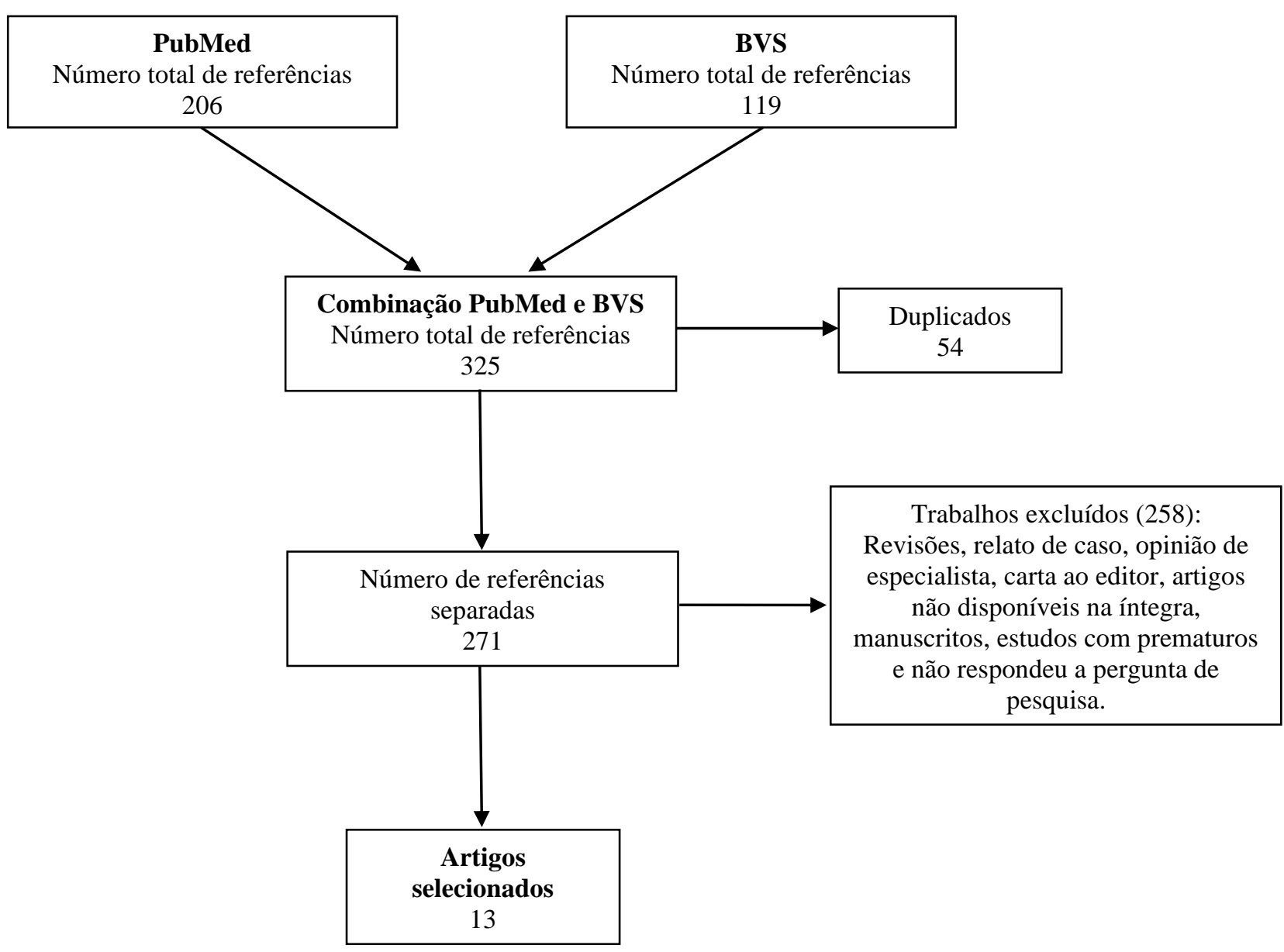

Fonte: Os autores

Entre os artigos selecionados, os anos de publicação variaram de 2011 a 2020, sendo os anos de 2016 e 2019, mais prevalentes. O idioma encontrado em todos os artigos foi o inglês e o periódico com maior número de publicações foram o The Journal of Allergy e o Clinical Immunology com $23 \%$

O quadro 2 mostra os artigos selecionados nessa revisão e seus respectivos 
autores, ano, desenho do estudo, nível de

evidência e conclusões.

Quadro 2 - Artigos incluídos na revisão.

\begin{tabular}{|c|c|c|}
\hline $\begin{array}{l}\text { Autores } \\
\text { Ano }\end{array}$ & $\begin{array}{l}\text { Tipo de } \\
\text { estudo/Nível } \\
\text { de evidência }\end{array}$ & Conclusão \\
\hline $\begin{array}{l}\text { Polos \& } \\
\text { Fletcher, } \\
2019\end{array}$ & $\begin{array}{l}\text { Estudo quase } \\
\text { experimental/ } \\
\text { IV }\end{array}$ & $\begin{array}{l}\text { A cesárea aumenta significativamente o risco de desenvolver alergia, } \\
\text { com significativas implicações qualitativas }\end{array}$ \\
\hline $\begin{array}{l}\text { Reyman, } \\
\text { et al., } \\
2019\end{array}$ & Coorte/IV & $\begin{array}{l}\text { Existe uma distinção na microbiota intestinal dos recém-nascidos de } \\
\text { parto normal e cesariana no primeiro ano de vida. Os bebês nascidos } \\
\text { por parto vaginal apresentam maior quantidade de Bifidobacterium e } \\
\text { redução de Enterococcus e Klebsiella spp. Ratificam que a composição } \\
\text { da microbiota intestinal na primeira semana de vida influencia no } \\
\text { número de infecções respiratórias. As crianças nascidas de cesariana } \\
\text { apresentam maior suscetibilidade para este tipo de agravo. }\end{array}$ \\
\hline $\begin{array}{l}\text { Begum, } \\
\text { et al., } \\
2019\end{array}$ & Coorte/IV & $\begin{array}{l}\text { Pode existir um pequeno aumento no risco de diabetes tipo I após a } \\
\text { cesariana. Pressupõe-se que a via de nascimento e a microbiota } \\
\text { neonatal adquirida pode não estar associada no desenvolvimento de } \\
\text { diabetes tipo I. }\end{array}$ \\
\hline $\begin{array}{l}\text { Marcotte, } \\
\text { et al., } \\
2018\end{array}$ & $\begin{array}{l}\text { Caso } \\
\text { Controle/IV }\end{array}$ & $\begin{array}{l}\text { Observou-se associação entre a cesariana e o risco de leucemia linfoide } \\
\text { agudo infantil. }\end{array}$ \\
\hline $\begin{array}{l}\text { Nagpa, et } \\
\text { al., } 2017\end{array}$ & Coorte/IV & $\begin{array}{l}\text { O uso de antibióticos na infância está associado ao aumento do risco de } \\
\text { doença atópica mais tardiamente. O estresse pré-natal e o precoce } \\
\text { podem alterar o perfil imunológico da criança e aumentar o risco de } \\
\text { asma e alergia. }\end{array}$ \\
\hline $\begin{array}{l}\text { Bosch, et } \\
\text { al., } 2017\end{array}$ & Coorte/IV & $\begin{array}{l}\text { A via de nascimento, o tipo de alimentação, o fato de ter de irmãos e o } \\
\text { uso recente de terapia antimicrobiana alteram o desenvolvimento da } \\
\text { microbiota, levando o RN a ser mais suscetível a infecções do trato } \\
\text { respiratório. }\end{array}$ \\
\hline $\begin{array}{l}\text { Kristens } \\
\& \\
\text { Herinkse } \\
\text { n, } 2016\end{array}$ & Coorte/IV & $\begin{array}{l}\text { A cesárea está associada à infecção e inflamação da mucosa e a lesões } \\
\text { idiopáticas juvenis. Esse resultado pode estar relacionado com a } \\
\text { resposta imune alterada induzida pela colonização microbiana } \\
\text { aberrante presente em crianças submetidas à referida via de } \\
\text { nascimento. }\end{array}$ \\
\hline $\begin{array}{l}\text { Curran, et } \\
\text { al., } 2016\end{array}$ & Coorte/IV & $\begin{array}{l}\text { O nascimento por cesariana é associada a um pequeno aumento do } \\
\text { risco de TDAH. }\end{array}$ \\
\hline $\begin{array}{l}\text { Wu, } \quad \text { et } \\
\text { al., } 2016\end{array}$ & Coorte/IV & $\begin{array}{l}\text { Exposições precoces, uso de antibiótico materno e infecção por ITU, } \\
\text { via de nascimento, uso de antibióticos infantis e número de irmãos } \\
\text { mais velhos em casa estão associados ao desenvolvimento da asma na }\end{array}$ \\
\hline
\end{tabular}




\begin{tabular}{|l|l|l|}
\hline & & infância de forma cumulativa. \\
\hline $\begin{array}{l}\text { Dogra, et } \\
\text { al., 2015 }\end{array}$ & Coorte/IV & $\begin{array}{l}\text { As bactérias que colonizam o corpo do RN ao nascer promovem um } \\
\text { efeito duradouro no sistema imunológico ou na função da barreira } \\
\text { intestinal, de acordo com a via de nascimento. Parto vaginal: } \\
\text { Bifidobacterium e Collinsella. Cesárea: Klebsiella, Enterobacteriaceae } \\
\text { e Streptococcus. Identificou-se uma colonização mais baixa ou mais } \\
\text { tardia por bifidobactérias, em bebês nascidos por cesariana, que são } \\
\text { consideradas bactérias ideais ao organismo do RN. }\end{array}$ \\
\hline $\begin{array}{l}\text { Penders, } \\
\text { et al., } \\
2013\end{array}$ & $\begin{array}{l}\text { Clinico } \\
\text { Controlado e e } \\
\text { randomizado }\end{array}$ & $\begin{array}{l}\text { Os resultados demonstram que a microbiota influencia no } \\
\text { desenvolvimento da dermatite atópica. A via de nascimento, a } \\
\text { amamentação e também a ordem de nascimento teve um efeito } \\
\text { significativo sobre a composição da microbiota. }\end{array}$ \\
\hline $\begin{array}{l}\text { Ajslev, et } \\
\text { al., 2011 }\end{array}$ & II & $\begin{array}{l}\text { Coorte/IV } \\
\text { II inoculação da microbiota materna por meio do parto vaginal em } \\
\text { comparação com a cesariana pode ser protetora do excesso de peso na } \\
\text { infância em meninos. Isso é constatado pela observação do aumento da } \\
\text { suscetibilidade ao excesso de peso em meninos nascidos por cesariana. }\end{array}$ \\
\hline $\begin{array}{l}\text { Nimwege } \\
\text { m et al., } \\
2011\end{array}$ & Coorte/IV & $\begin{array}{l}\text { O modo e o local do parto afetam a composição da microbiota } \\
\text { gastrointestinal que, subsequentemente, provoca o risco de } \\
\text { manifestações atópicas. O atraso na colonização por bactérias } \\
\text { benéficas pode interferir no desenvolvimento de doenças imunológicas. }\end{array}$ \\
\hline
\end{tabular}

Fonte; Os autores.

\section{DISCUSSÃO}

Acredita-se que existem três mecanismos envolvidos durante o parto e que esses estão relacionados ao desenvolvimento do sistema imunológico. São eles: o estresse adaptativo gerado pelo trabalho de parto e pelo parto vaginal, as alterações epigenéticas e o microbioma que o RN é exposto ao nascer 16 .

O estresse gerado pelas contrações e posterior hipóxia no feto aumentam os níveis de catecolaminas e corticoides que desempenham um papel na maturação de órgãos, como intestinos e pulmões. Os níveis dessas substâncias são comprovadamente menores em bebês nascidos por cesariana. Durante o período pré-natal e nos primeiros anos após o nascimento, existe alta sensibilidade em alterações epigenéticas e modificações de fenótipo. Esse processo afeta diretamente a produção de anticorpos, a regulação da glicose e da resposta a alérgenos e é o motivo de maior risco de distúrbios metabólicos, como obesidade e diabetes mellitus em bebês nascidos por cesariana ${ }^{16}$. Entretanto, um estudo de coorte refere que existem inconsistência sobre a associação entre a cesariana e o risco de diabetes do tipo 
I. Os seus resultados corroboram com outros estudos de coorte realizados na Suécia e Noruega em que encontrou um risco nulo ou pequena associação entre esta via de nascimento e o agravo mencionado ${ }^{17}$.

No entanto, ratifica-se que o microbioma adquirido depende da via de nascimento. Os bebês nascidos por via vaginal são colonizados por bactérias do canal de parto da mãe, e os nascidos por cesárea, por microorganismos presentes no ambiente e na pele. Esses agentes são predominantemente patogênicos, e têm maior e menor quantidade de Clostridium difficile (microorganismo patogênico) e de Bifidobactéria (microorganismo protetor), respectivamente ${ }^{16,18}$. Em relação ao parto vaginal, estudo mostrou que bebês nascidos por essa via abrigam comunidades bacterianas muito semelhantes às de suas mães, dominadas por microorganismos protetores para o RN, como Lactobacillus, Prevotella ou Sneathia spp ${ }^{19}$. Além disso, a criança que nasce por cesárea possui uma microbiota um tanto instável quando comparada à dos bebês nascidos por via vaginal ${ }^{20,21}$.

Nesta mesma direção, outra pesquisa, de coorte, com 120 crianças, sendo 74 nascidas de parto vaginal e 46 por cesariana, demonstrou a diferença da microbiota intestinal entre os bebês, conforme a via de nascimento durante o primeiro ano de vida, independente do uso de antibióticos maternos.
Ela encontrou a propagação da microbiota intestinal da mãe para o $\mathrm{RN}$ de parto normal e o desenvolvimento de uma microbiota mais estável no começo da vida, principalmente na primeira semana, quando comparado aqueles que nasceram por cesárea. Os primeiros apresentaram maior quantidade de Bifidobactéria e a redução de Enterecocos e Klebsiela que são potencialmente patogênicos, conforme mencionado. Esta composição está associada ao número de infecções respiratórias que uma criança terá no primeiro ano de vida ${ }^{9}$. Propõe-se que a cesariana contribui para uma microbiota intestinal mais adversa para o recém-nascido 9,22 .

Em uma revisão, os autores demonstram que a microbiota intestinal geralmente possui duas transições na infância. A primeira ocorre logo após o nascimento, durante a lactação e possui o predomínio por Bifidobacterias. A segunda transição ocorre durante o período do desmame com a introdução alimentar e a microbiota passa a ser mais complexa constituída principalmente por bacteroides e firmicutes. Essas alterações continuam até os três anos. Sobrelevam que o estabelecimento precoce da microbiota intestinal é influenciado pela via de nascimento, aleitamento, uso de antibióticos, desmame e introdução alimentar. Essa colonização inicial tem efeitos no desenvolvimento e na fisiologia do sistema 
imunológico e afeta a saúde na vida adulta. Uma alteração nestes processos pode causar doenças imunológicas, como alergias alimentares, dermatite atópica e asma ${ }^{23}$.

Deste modo, entende-se que a composição desta microbiota e os primeiros colonizadores intestinal influenciam no sistema imunológico e no desenvolvimento de determinadas patologias ao longo da vida. Porém, sabe-se pouco sobre os processos envolvidos e como o microbioma são adquiridos ${ }^{24}$. Depreende-se que é fundamental evidenciar o impacto da microbiota adquirida do RN na saúde infantil, conforme a via de nascimento descrita na produção de conhecimento. A seguir, a discussão é apresentada em três categorias de análise: as doenças na infância e a cesariana; Fatores de risco associados a alterações na microbiota do RN; e Fatores protetores associados a alterações na microbiota do RN.

\section{As doenças na infância e a cesariana}

As doenças relacionadas à cesariana mais citadas nos artigos desta revisão foram: doenças atópicas: eczema, alergia alimentar e dermatite 25-29; doenças inflamatórias intestinais: doença de Crohn, enterocolite, síndrome do intestino irritável e colite ulcerosa ${ }^{21,27-30}$; doenças respiratórias: asma e laringite 11,26,27,31-33; doenças imunes: artrite idiopática juvenil e leucemia linfoblástica aguda; transtornos mentais: TDAH; e doenças metabólicas como a obesidade ${ }^{34,35}$.

Autores observaram que a colonização por clostridium difficle, no primeiro mês de vida, foi associada a chiado e à asma, dos 6 aos 7 anos de idade ${ }^{26}$. Constatou-se, em outro estudo, que os bebês nascidos por cesárea tiveram $34 \%$ mais chances de desenvolvê-la na infância quando comparados aos nascidos por parto vaginal ${ }^{33}$. A asma, distúrbio inflamatório crônico das vias aéreas, é causada por fatores genéticos e ambientais. A ação patogênica do Clostridium nessa doença não é bem estabelecida pela literatura. Em contrapartida, pesquisa aponta que alguns microorganismos protetores, como é o caso das bifidobactérias, são capazes de prevenir o desenvolvimento da mesma ${ }^{36}$. Mais estudos são necessários para determinar os impactos microbianos no epitélio pulmonar.

Corrobora-se com estes resultados, onde um estudo de coorte evidenciou que $\mathrm{RN}$ nascidos por cesariana apresentaram uma tendência maior de infecções respiratórias e o uso de antibióticos quando comparados aos nascidos por parto vaginal no primeiro ano de vida. Acrescentam que a composição da microbiota intestinal instável do $\mathrm{RN}$ de cesárea é independente da exposição pré-natal a antibióticos, tempo de hospitalização e do tipo de alimentação do mesmo (aleitamento materno e leite artificial). Estes achados fornecem evidências de uma associação das 
alterações induzidas pela via de nascimento na microbiota intestinal do bebê e a suscetibilidade a doenças infecciosas ${ }^{9}$.

As doenças inflamatórias intestinais aparecem com frequência nos artigos selecionados. Afirma-se que a alta abundância de Clostridium difficile é considerada uma das principais ameaças que causam infecções gastrintestinais graves durante a infância ${ }^{30}$. Ao chegar ao cólon, os esporos do clostridium se transformam em suas formas vegetativas e liberam enterotoxinas causadoras de colite e de sintomas diarréicos, denominadas enterotoxina TcdA e citotoxina TcdB. As toxinas produzidas pela bactéria são capazes de se ligarem a receptores celulares específicos, causando alterações no citoesqueleto da actina, ruptura da barreira epitelial, apoptose e resposta inflamatória no hospedeiro ${ }^{37}$.

Outras doenças gastrintestinais foram citadas, como doença de Crohn, enterocolite, síndrome do intestino irritável, colite ulcerosa 21,27-29. Entretanto, em uma metanálise, não se observou diferença significativa entre o risco de desenvolvimento de doença intestinal nos bebês nascidos por cesárea ${ }^{38}$. Em uma pesquisa, quase experimental, aponta que a cesariana aumenta significativamente a alergia alimentar com implicações qualitativas ${ }^{22}$. Acrescenta-se que o ambiente é a principal fonte de contaminação de Clostridium perfringens, microorganismo enterotóxico, que causa doenças gastrintestinais em bebês que nascem por cesárea e, por consequência, permanecem mais tempo hospitalizados após o nascimento ${ }^{21}$.

O recém-nascido nascido por cesariana possui maior propensão para adquirir sobrepeso em todas as fases da vida, independente do peso ao nascer ou dos fatores de risco para obesidade. $\mathrm{O}$ procedimento altera o microbioma por meio de interação micróbio-hospedeiro e modifica o metabolismo lipídico e as respostas inflamatórias do $\mathrm{RN}^{32}$. Em outro estudo, não foi encontrada associação significativa entre a via de nascimento e o risco de sobrepeso infantil, no entanto, identificou que existe um aumento do risco de sobrepeso em meninos nascidos por cesárea ${ }^{18}$. Percebe-se que existem divergências nas publicações e são necessários mais estudos que discutam a obesidade em relação à via de nascimento.

A dermatite atópica também foi citada como uma complicação que pode ocorrer na cesariana. A composição da microbiota e o risco de desenvolvimento da referida dermatite tiveram associação positiva, sendo a presença do clostridium relacionada ao desenvolvimento do agravo no bebê ${ }^{25}$. Podese inferir que mais estudos são necessários para comprovar a associação da cesárea ao surgimento da dermatite na primeira infância.

Somente em um artigo, a cesariana foi associada ao risco de TDAH. Demonstrou-se 
que o nascimento por cesárea aumentou em $14 \%$ a $16 \%$ o risco de desenvolvimento de TDAH em comparação ao parto vaginal, principalmente nos casos das de emergência 35. Esses resultados demonstram que é fundamental compreender os mecanismos envolvidos nessa associação, principalmente em virtude da incidência elevada de cesariana e de TDAH.

Em outro trabalho, foi analisada a relação da via de nascimento com a ocorrência de leucemia infantil. Verificou-se que a cesárea foi associada a um elevado risco de se adquirir leucemia do tipo linfoblástica aguda (LLA) ${ }^{34}$. A resposta ao estresse do parto aumenta as concentrações de cortisol e de catecolaminas em 1,5 a 3 vezes em bebês nascidos por via vaginal em comparação aos nascidos por cesárea, antes do trabalho de parto. Esse aumento imita os efeitos antileucêmicos dos glicocorticosteróides que são usados no tratamento da leucemia infantil. $\mathrm{O}$ estudo sugere que a exposição a níveis elevados de cortisol, durante o trabalho de parto e parto, tem um importante papel na redução do risco de LLA para lactentes que abrigam células pré-leucêmicas que surgiram no útero. $\mathrm{O}$ mesmo estudo não encontrou relação com os outros tipos de leucemia ${ }^{34}$.

Fatores de risco associados a alterações na microbiota do $\mathbf{R N}$
Alguns estudos destacaram o uso de antibióticos como um fator que influencia negativamente no desenvolvimento da microbiota saudável do bebê, tanto quando usados na gestação quanto na primeira infância $18,21,32,33$. Afirma-se que o uso dos antimicrobianos durante a gestação está associado a uma diversidade microbiana reduzida, principalmente, de microorganismos protetores, como lactobacilos e bifidobactérias ${ }^{32}$. Uma revisão sistemática observou que o uso da ampicilina pode substituir enterobactérias benéficas por enterobactérias resistentes ao antibiótico, desfavorecendo a microbiota do $\mathrm{RN}^{39}$. Outra, ratificou que os bebês que foram tratados com cefalexina tiveram a diversidade da microbiota reduzida, especificamente, de bifidobactérias, e crescimento excessivo de Enterococcus e Enterobacteriaceae, no primeiro mês de vida, quando comparados aos bebês não tradados ${ }^{29}$.

A prematuridade foi citada como outro fator de risco ${ }^{25}$. Demonstram-se que as bactéricas benéficas (bifidobactérias e lactobacilos), comumente encontradas em bebês a termo, raramente foram detectadas nas fezes de bebês prematuros. Além disso, salientam-se que os primeiros colonizadores do intestino prematuro foram organismos potencialmente patogênicos, como Escherichia coli, Streptococcus, Klebsiella, Clostridium, Enterococcus e Staphylococcus 
29. Verificou-se que bebês prematuros, com peso de nascimento inferior a $2500 \mathrm{~g}$, eram colonizados por bactérias protetoras, com menos frequência ${ }^{25}$.

\section{Fatores protetores associados às alterações} na microbiota do $\mathbf{R N}$

Existem outras associações relevantes para o desenvolvimento da microbiota do RN, tais como amamentação, lugar de nascimento (parto domiciliar versus hospitalar), existência de irmãos mais velhos e risco alto de infecção do trato urinário na gestação ${ }^{11,21,30,34}$.

A amamentação foi citada como fator de proteção para o bebê ${ }^{9,11,21}$. Foi observado que crianças amamentadas por mais de seis meses de vida tiveram grandes diferenças na composição do microbioma, com 31 semanas, quando comparadas às que foram amamentadas por um período menor, e que a amamentação também tem potencial para a redução do risco de doenças imunomediadas e metabólicas ${ }^{25,40}$.

Em outro trabalho, comparou-se, durante a infância, por meio da via de nascimento e da alimentação, o transporte intestinal de Clostridium perfringens $\alpha$ toxigênico e enterotoxigênico com o transporte de outros microorganismos intestinais. Foi evidenciado que entre os bebês nascidos por cesárea, os que foram amamentados exclusivamente apresentaram níveis mais baixos de $C$. perfringens e níveis mais altos de microorganismos protetores, como lactobacilos e bifidobactérias, tendo a amamentação um importante papel na prevenção de doenças neonatais ${ }^{21}$.

Ademais, em outra pesquisa identificou-se que metade dos bebês nascidos por parto vaginal, com 31 semanas de idade gestacional, foi espontaneamente colonizada com lactobacilos, e que a porcentagem desta colonização nos nascidos por cesariana foi menor (37,5\%). O estudo destaca a amamentação como um importante fator de proteção, pois o leite materno possui compostos bioativos, incluindo imunoglobulinas, citocinas, hormônios, enzimas e micróbios que contribuem para o desenvolvimento da microbiota do bebê ${ }^{25}$.

Outro trabalho demonstrou a importância da amamentação, contudo, destaca a influência da via de nascimento, sobretudo a vaginal. Ele evidenciou que a estimulação da amamentação em mulheres submetidas à cesariana ou o avanço nas fórmulas de leite não foram capazes de corrigir a colonização por Bifidobacterium, pois RN nascidos de cesárea e amamentados apresentaram esta bactéria em menor quantidade quando comparados aos RNs de parto vaginal e que usaram o leite artificial.

Entre outros fatores, destacam-se o fato de ter irmãos mais velhos e o local do 
parto vaginal. No primeiro, a colonização por Clostridium diminuiu em relação ao aumento do número de irmãos, e o parto vaginal domiciliar reduziu o risco de eczema, de sensibilização a alérgenos alimentares e de asma, quando comparado com o hospitalar ${ }^{25-}$ 27.

\section{CONCLUSÃO}

Esta revisão incluiu 13 artigos internacionais que tratam da influência do tipo de nascimento e a microbiota adquirida na saúde infantil. É unânime entre os autores que a via de nascimento exerce influência na composição da microbiota do RN. Alguns outros fatores são destacados em parte dos estudos, como a amamentação, higiene, vínculo mãe e filho, tempo de hospitalização, existência de irmãos mais velhos, local de nascimento, uso de medicamentos na gestação e na infância, entre outros.

Os estudos contemplados verificaram fatores que podem interferir no desenvolvimento de doenças na infância em crianças nascidas por cesariana, como asma, dermatite, obesidade, doença intestinal, metabólica e até mesmo alguns tipos de cânceres. Desse modo, contribui para a discussão dessa temática e amplia o conhecimento dos profissionais da saúde, das mulheres e seus familiares envolvidos no 1 . processo de gestar, parir e maternar. Esperamse mais experiências positivas e escolhas informadas e conscientes em relação à via de nascimento.

Ademais, o aumento das doenças na infância é um sério problema de saúde pública com repercussões imediatas na vida das crianças, custos econômicos para as famílias, governo e impactos a longo prazo na vida adulta. Com isso, é necessária a divulgação dessas informações tanto para as mulheres, durante o pré-natal ou por meio de grupos educativos, como para os profissionais da saúde, na realização de cursos de capacitações. Além disso, essa temática deve ser discutida no meio acadêmico para agregar mais conhecimento aos estudantes sobre a importância da realização da cesárea somente quando existirem indicações reais.

No entanto, apesar das evidências apresentadas, mais estudos precisam ser realizados a fim de reduzir as lacunas relacionadas à saúde infantil e sua associação com a composição da microbiota conforme a via de nascimento. Além do que, existem poucos estudos que conseguiram excluir os fatores de confusão. Sabe-se as doenças na infância podem estar associadas a outros fatores como história familiar, ambiente, entre outros.

\section{REFERÊNCIAS}

Wells JC, Wibaek R, Poullas M. Global epidemiology of use of and disparities in caesarean sections. Lancet [Internet]. 2019;394(10192):24-5. Available at: 
https://www.thelancet.com/journals/lancet/arti cle/PIIS0140-6736(19)30715-9/fulltext

2. Bonamigo EL, Braga DC, Andreis B, Rossi SA. Fatores associados à realização de parto cesáreo em mulheres brasileiras uma análise 8 . da Pesquisa Nacional de Saúde, PNS 2013. Rev da Amrigs [Internet]. 2020;64(August):10-7. Available at: https://www.researchgate.net/profile/Camila_ Hood/publication/343994787_Sistemas_nacio nais_de_transplante_-

_Brasil_versus_Holanda_aprimoramento_par a_garantia_da_continuidade_da_vida/links/5f 4d09c6458515a88b999003/Sistemasnacionais-de-transplante-Brasil-ve

3. OMS. Declaração da OMS sobre Taxas de Cesáreas. Hum Reprod Program [Internet]. 2015;1-8. Available at: 10 http://www.who.int/about/ licensing/copyright_form/en/index.html).

4. BRASIL. Informações de saúde: estatística vitais. Sistema de Informações sobre Nascidos Vivos (SINASC), 2013. [Internet]. Ministério da Saúde. 2014. Available at: http://tabnet.datasus.gov.br/cgi/deftohtm.exe? sinasc/cnv/nvuf.def

5. BRASIL. Taxas de partos cesáreos por operadora de plano de saúde. Informações e Avaliações de Operadoras, 2013. [Internet]. Agência Nacional de Saúde Suplementar. 2013. Available at: https://www.ans.gov.br/planos-de-saude-eoperadoras/informacoes-e-avaliacoes-deoperadoras/taxas-de-partos-cesareos-poroperadora-de-plano-de-saude

6. Oliveira RR de, Melo EC, Novaes ES, Ferracioli PLRV, Mathias TA de F. Factors associated to Caesarean delivery in public and private health care systems. Rev da Esc Enferm [Internet]. 2016;50(5):733-40. Available at: https://www.scielo.br/scielo.php?pid=S008062342016000500733\&script=sci_arttext

7. Patah LEM, Malik AM. Models of child birth care and cesarean rates in different countries. Rev Saude Publica [Internet].
2011;45(1):185-94. Available at: https://www.scielo.br/scielo.php?pid=S003489102011000100021\&script=sci_arttext\&tlng $=\mathrm{es}$

Kristensen K, Henriksen L. Cesarean section and disease associated with immune function. J Allergy Clin Immunol [Internet]. 2015;137(2):587-90. Available at: https://pubmed.ncbi.nlm.nih.gov/26371844/

Reyman M, Houten MA van, Baarle D van, Bosch AATM, Man WH, Chu MLJN, et al. Impact of delivery mode-associated gut microbiota dynamics on health in the first year of life. Nat Commun [Internet]. 2019;10(1):1-12. Available at: https://www.nature.com/articles/s41467-01913014-7

0. Dominguez-Bello MG, De Jesus-Laboy KM, Shen N, Cox LM, Amir A, Gonzalez A, et al. Partial restoration of the microbiota of cesarean-born infants via vaginal microbial transfer. Nat Med. 2016;22(3):250-3.

11. Bosch AATM, Levin E, van Houten MA, Hasrat R, Kalkman G, Biesbroek G, et al. Development of Upper Respiratory Tract Microbiota in Infancy is Affected by Mode of Delivery. EBioMedicine [Internet]. 2016;9:336-45. Available at: http://dx.doi.org/10.1016/j.ebiom.2016.05.031

12. Biasucci G, Benenati B, Morelli L, Bessi E, Boehm G. Cesarean delivery may affect the early biodiversity of intestinal bacteria. J Nutr [Internet]. 2008;138(9):1796-800. Available at:

https://pubmed.ncbi.nlm.nih.gov/18716189/

13. Sousa LMM de, Marques-Vieira CMA, Severino SSP, Antunes AV. A metodologia de revisão integrativa da literatura em enfermagem. Rev Investig em Enferm [Internet]. 2017;21(2):17-26. Available at: http://www.sinaisvitais.pt/images/stories/Rie/ RIE21.pdf\#page $=17$

14. Mendes KDS, Silveira RC de CP, Galvão CM. Revisão integrativa: método de pesquisa para a incorporação de evidências na saúde e na enfermagem. Texto Context - Enferm. 
2008;17(4):758-64.

15. Ursi ES, Gavão CM. Prevenção de lesões de 23. pele no perioperatório: revisão integrativa da literatura. Rev Lat Am Enfermagem [Internet]. 2006;14(1):2-127. Available at: https://www.scielo.br/pdf/rlae/v14n1/v14n1a1 7.pdf

16. J H, P P, J Z. The impact of physiological 24. peripartal stress on the lifelong health of newborn. Bratisl lek??rske List [Internet]. 2017;116(5):324-7. Available at: https://pubmed.ncbi.nlm.nih.gov/28664740/

17. Begum M, Pilkington R, Chittleborough C, Lynch J, Penno M, Smithers L. Caesarean section and risk of type 1 diabetes: whole-ofpopulation study. Diabet Med. 25. 2019;36(12):1686-93.

18. Ajslev TA, Andersen CS, Gamborg $\mathrm{M}$, Sørensen TIA, Jess T. Childhood overweight after establishment of the gut microbiota: the role of delivery mode, pre-pregnancy weight and early administration of antibiotics. Int $\mathrm{J}$ Obes. 2011;35(4):522-9.

19. Dominguez-Bello MG, Costello EK, Contreras M, Magris M, Hidalgo G, Fierer N, et al. Delivery mode shapes the acquisition and structure of the initial microbiota across multiple body habitats in newborns. Proc Natl Acad Sci U S A. 2010;107(26):11971-5.

20. Dogra S, Sakwinska O, Soh SE, Ngom-Bru C, Brück WM, Berger B, et al. Dynamics of infant gut microbiota are influenced by delivery mode and gestational duration and are associated with subsequent adiposity. MBio. 2015;6(1):1-9.

21. Nagpal R, Tsuji H, Takahashi T, Nomoto K, Kawashima K, Nagata S, et al. Gut dysbiosis following c-section instigates higher colonisation of toxigenic clostridium 29. perfringens in infants. Benef Microbes. 2017;8(3):353-65.

22. Polos J, Fletcher J. Caesarean section and children's health: A quasi-experimental 30. design. Author Manuscr [Internet]. 20119;73(3):353-68. Available at: https://pubmed.ncbi.nlm.nih.gov/31271341/

Tanaka M, Nakayama J. Development of the gut microbiota in infancy and its impact on health in later life. Allergol Int [Internet]. 2017;66(4):515-22. Available at: https://www.sciencedirect.com/science/article /pii/S1323893017301119

Maqsood R, Rodgers R, Rodriguez C, Handley SA, Ndao IM, Tarr PI, et al. Discordant transmission of bacteria and viruses from mothers to babies at birth. Microbiome [Internet]. 2019;7(1):1-13. Available at: https://microbiomejournal.biomedcentral.com /articles/10.1186/s40168-019-0766-7

Penders J, Gerhold K, Stobberingh EE, Thijs C, Zimmermann $\mathrm{K}$, Lau $\mathrm{S}$, et al. Establishment of the intestinal microbiota and its role for atopic dermatitis in early childhood. J Allergy Clin Immunol [Internet]. 2013;132(3):601-607.e8. Available at: http://dx.doi.org/10.1016/j.jaci.2013.05.043

Van Nimwegen FA, Penders J, Stobberingh EE, Postma DS, Koppelman GH, Kerkhof M, et al. Mode and place of delivery, gastrointestinal microbiota, and their influence on asthma and atopy. J Allergy Clin Immunol. 2011;128(5):948-955.e3.

Saavedra JM, Dattilo AM. Early Development of Intestinal Microbiota. Implications for Future Health. Gastroenterol Clin North Am [Internet]. 2012;41(4):717-31. Available at: http://dx.doi.org/10.1016/j.gtc.2012.08.001

Kozyrskyj A, Bahrein S, MB. Early life exposures: impact on asthma and allergic disease. Curr opin allergy clin immunol. 2011;11:400-6.

Li M, Wang M, Donovan SM. Early development of the gut microbiome and immune-mediated childhood disorders. Semin Reprod Med. 2014;32(1):74-86.

Rutayisire E, Huang K, Liu Y, Tao F. The mode of delivery affects the diversity and colonization pattern of the gut microbiota 
during the first year of infants' life: A 38 . systematic review. BMC Gastroenterol [Internet]. 2016;16(1):1-12. Available at: http://dx.doi.org/10.1186/s12876-016-0498-0

31. A S, J S, K B, H B. Cesarean Section and Chronic Immune Disorders. Pediatrics 39. [Internet]. 2015;1:93-8. Available at: https://pediatrics.aappublications.org/content/ 135/1/e92?sso=1\&sso_redirect_count $=1 \& \mathrm{nfst}$ atus $=401 \&$ nftoken $=00000000-0000-0000$ 0000-

000000000000\&nfstatusdescription=ERROR $\% 3 \mathrm{~A}$ No local token

32. Montoya-Williams D, Lemas DJ, Spiryda L, Patel K, Carney OO, Neu J, et al. The Neonatal Microbiome and Its Partial Role in Mediating the Association between Birth by Cesarean Section and Adverse Pediatric Outcomes. Neonatology. 2018;114(2):10311.

33. Wu P, Feldman AS, Rosas-Salazar C, James $\mathrm{K}$, Escobar G, Gebretsadik T, et al. Relative importance and additive effects of maternal and infant risk factors on childhood asthma. PLoS One. 2016;11(3):1-16.

34. Marcotte EL, Richardson MR, Roesler MA, Spector LG. Cesarean delivery and risk of infant Leukemia: A report from the children's oncology group. Cancer Epidemiol Biomarkers Prev. 2018;27(4):473-8.

35. Curran EA, Khashan AS, Dalman C, Kenny LC, Cryan JF, Dinan TG, et al. Obstetric mode of delivery and attentiondeficit/hyperactivity disorder: A siblingmatched study. Int $\mathbf{J}$ Epidemiol [Internet]. 2016;45(2):532-42. Available at: https://academic.oup.com/ije/article/45/2/532/ 2572619? $\operatorname{login}=$ true

36. Campos HS. Papel do microbioma na resposta imune e na asma. Arq Asma, Alerg e Imunol. 2018;2(2):238-46.

37. Novo LMG, Tosta VM de M, Oliveira RCF de, Simioni PU. Os Efeitos dos Probióticos nas Infecções Recorrentes por Clostridium Difficile. 2016;
Bruce A, Black M, Bhattacharya S. Mode of delivery and risk of inflammatory bowel disease in the offspring: Systematic review and meta-analysis of observational studies. Inflamm Bowel Dis. 2014;20(7):1217-26.

Yang I, Corwin EJ, Brennan PA, Jordan S, Murphy JR, Dunlop A. The infant microbiome: Implications for infant health and neurocognitive development. Nurs Res [Internet]. 2016;65(1):76-88. Available at: https://www.ncbi.nlm.nih.gov/pmc/articles/P MC4681407/

40. Rautava S, Luoto R, Salminen S, Isolauri E. Microbial contact during pregnancy, intestinal colonization and human disease. Nat Rev Gastroenterol Hepatol [Internet]. 2012;9(10):565-76. Available at: https://www.nature.com/articles/nrgastro.201 2.144

\section{Autor correspondente ${ }^{\#}$}

Lilian Fernandes Arial Ayres, Av Ph Rolphs, s/n, Campus Viçosa, Viçosa/MG, CEP 36570000, telefone: (31) 984374997, E-mail: lilian.ayres@ufv.br

Submissão: 2021-06-01

Aprovado: 2021-08-16 